\title{
State Feedback Sliding Mode Controller Design for Human Swing Leg System
}

\author{
Hazem I. Ali \\ Control and Systems Eng. Dep. \\ University of Technology \\ hazemcontrol2001@yahoo.com \\ Received: 07-June-2017 Revised: 18-Oct.-2017 Accepted: 18-Dec.-2017 \\ http://doi.org/10.29194/NJES21010051
}

\begin{abstract}
In this paper, the robustness properties of sliding mode control (SMC) which is designed to produce a dynamic output feedback controller to achieve robustness for trajectory tracking of the nonlinear human swing leg system is presented. The human swing leg represents the support of human leg or the humanoid robot leg which is usually modeled as a double pendulum. The thigh and shank of a human leg will respect the pendulum links, hip and knee will connect the upper body to thigh and then shank respectively. The total moments required to move the muscles of thigh and shank are denoted by two external (servomotors) torques applied at the hip and knee joints. The mathematical model of the system is developed. The results show that the proposed controller can robustly stabilize the system and achieve a desirable time response specification.
\end{abstract}

Keywords: Human Swing Leg, Sliding Mode Control (SMC), full state feedback controller, uncertain system.

\section{Introduction}

Human movement represents the translation of human body from one place to another. It is considered from three perspectives of walking, jogging, and running gaits. One of the main gaits of movement is walking and happens more frequently than the other ones [1]. The complicated physics of the leg locomotion make it one of the most complex motions in the support of human leg or in the humanoid robot leg due to its complicated physics [2]. There are a large number of people who lost their walking ability due to neurological injuries, such as spinal cord injury and stroke, which result in motor-incomplete gait [3].

The joints of the body are the connections of system devices for each other. The relative motions of these devices are determined with respect to each other through the joint. This type of joints is referring to as self-impact joints. Joint self-impact phenomenon happens between shank and thigh at knee joint in specific times. Figure 1 shows the joint self-impact, when shank and thigh are aligned [1].

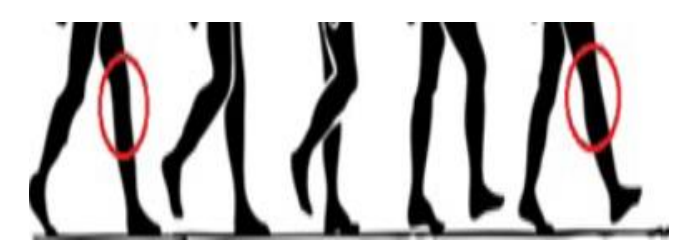

Figure 1: Joint self-impact in a complete period of walking, when shank and thigh are aligned [1].

The concept of Sliding Mode Control (SMC) is known for robustness and stability. It can be achieved by changing the controller structure. In the SMC, the system state trajectory is forced to move along a chosen stable manifold, called the sliding manifold, in the state space. The sliding manifold is always chosen in such a manner that derived control law guarantees the system stability [4]. SMC has proven to be an effective robust control strategy. Many important results have been reported in the literature on sliding mode control of different classes of systems. Sliding mode controller has been applied for uncertain systems, time delay systems, stochastic systems, and switched hybrid systems [5].

The main goal of this paper is to design the sliding mode controller to stabilize the human swing leg system and achieve a desirable tracking.

\section{System Mathematical Model}

In support human leg and humanoid robot leg, swing leg system is largely achieved by the motion of the hip and knee while the ankle contribution can be neglected. The human swing leg usually modeled as a double pendulum with the thigh and shank represented as two links with their masses $\mathrm{m}_{1}, \mathrm{~m}_{2}$. The lengths of thigh and shank are $l_{1}, l_{2}$ and the unconstrained double pendulum is shown in Figure 2. In this figure, $\theta_{1}$ and $\theta_{2}$, denote the hip and knee rotation angles, respectively. $\tau_{1}$ and $\tau_{2}$ are the applied external torques that move the thigh and shank links $[1,2$, $6]$. 


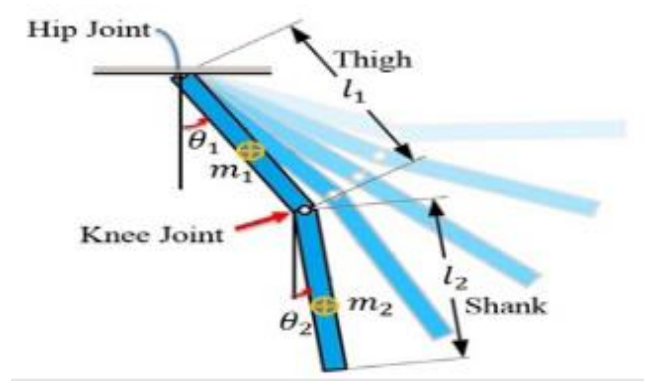

Figure 2: Schematics human swing leg [6]. [1]:

The dynamic of the system based on Lagrange's method can be represented by the following equations

$$
\begin{aligned}
& \frac{\left(m_{1}+3 m_{2}\right)}{3} l_{1}^{2} \ddot{\theta}_{1}+\frac{m_{2} l_{1} l_{2} \ddot{\theta}_{2}}{2} \cos \left(\theta_{1}-\theta_{2}\right)+\frac{m_{2} l_{1} l_{2} \dot{\theta}_{2}^{2}}{2} \sin \left(\theta_{1}-\theta_{2}\right)+\frac{\left(m_{1}+2 m_{2}\right)}{2} g l_{1} \sin \theta_{1}=\tau_{1} \\
& \frac{m_{2}}{3} l_{2}^{2} \ddot{\theta}_{2}+\frac{m_{2} l_{1} l_{2} \ddot{\theta}_{1}}{2} \cos \left(\theta_{1}-\theta_{2}\right)-\frac{m_{2} l_{1} l_{2} \dot{\theta}_{1}^{2}}{2} \sin \left(\theta_{1}-\theta_{2}\right)+\frac{m_{2}}{2} g l_{2} \sin \theta_{2}=\tau_{2}
\end{aligned}
$$

The dynamical equations of a swing leg, which is modeled as unconstrained double pendulum, may be rewritten in the following form $[1,6]$ :

$$
M(\theta) \ddot{\theta}+C(\theta, \dot{\theta}) \dot{\theta}+G_{t}(\theta)=\tau
$$

where $\theta, \dot{\theta}, \ddot{\theta}$ are $2 \times 1$ vectors of joint angles, joint angular velocities, and joint angular accelerations, respectively; $M(\theta)$ is $2 \times 2$ symmetric positive definite inertia matrix; $C(\theta, \dot{\theta}) \dot{\theta}$ is $2 \times 1$ vector of Coriolis; $G_{t}(\theta)$ is $2 \times 1$ vector of gravitational torques and $\tau$ is $2 \times 1$ vector of actuator joint torques, where

$$
\begin{aligned}
& M(\theta)=\left[\begin{array}{cc}
\frac{\left(m_{1}+3 m_{2}\right)}{3} l_{1}^{2} & \frac{m_{2} l_{1} l_{2}}{2} \cos \left(\theta_{1}-\theta_{2}\right) \\
\frac{m_{2} l_{1} l_{2}}{2} \cos \left(\theta_{1}-\theta_{2}\right) & \frac{m_{2}}{3} l_{2}^{2}
\end{array}\right], G_{t}(\theta)=\left[\begin{array}{c}
\frac{\left(m_{1}+2 m_{2}\right)}{2} g l_{1} \sin \theta_{1} \\
\frac{m_{2}}{2} g l_{2} \sin \theta_{2}
\end{array}\right] \\
& C(\theta, \dot{\theta})=\left[\begin{array}{cc}
0 & \frac{m_{2} l_{1} l_{2} \dot{\theta}_{2}}{2} \sin \left(\theta_{1}-\theta_{2}\right) \\
\frac{-m_{2} l_{1} l_{2} \dot{\theta}_{1}}{2} \sin \left(\theta_{1}-\theta_{2}\right) & 0
\end{array}\right], \tau=\left[\begin{array}{l}
\tau_{1} \\
\tau_{2}
\end{array}\right]
\end{aligned}
$$

Simplifying equations (1) and (2) yields:

$$
\begin{aligned}
& \ddot{\theta}_{1}=\frac{K_{4}\left(\tau_{1}-K_{2} \dot{\theta}_{2}^{2} \sin \left(\theta_{1}-\theta_{2}\right)-K_{3} \sin \theta_{1}\right)-K_{2} \cos \left(\theta_{1}-\theta_{2}\right)\left(\tau_{2}+K_{2} \dot{\theta}_{1}^{2} \sin \left(\theta_{1}-\theta_{2}\right)-K_{5} \sin \theta_{2}\right)}{\left(K_{1} K_{4}-K_{2}^{2} \cos \left(\theta_{1}-\theta_{2}\right)^{2}\right.} \\
& \ddot{\theta}_{2}=\frac{K_{1}\left(\tau_{2}+K_{2} \dot{\theta}_{1}^{2} \sin \left(\theta_{1}-\theta_{2}\right)-K_{5} \sin \theta_{2}\right)-K_{2} \cos \left(\theta_{1}-\theta_{2}\right)\left(\tau_{1}-K_{2} \dot{\theta}_{2}^{2} \sin \left(\theta_{1}-\theta_{2}\right)-K_{3} \sin \theta_{1}\right)}{\left(K_{1} K_{4}-K_{2}^{2} \cos \left(\theta_{1}-\theta_{2}\right)^{2}\right.}
\end{aligned}
$$

where

$$
K_{1}=\frac{\left(m_{1}+3 m_{2}\right)}{3} l_{1}^{2}, K_{2}=\frac{m_{2} l_{1} l_{2}}{2}, K_{3}=\frac{\left(m_{1}+2 m_{2}\right)}{2} g l_{1}, K_{4}=\frac{m_{2}}{3} l_{2}^{2}, K_{5}=\frac{m_{2}}{2} g l_{2}
$$

Assume the state variables are:

$x_{1}=\theta_{1}$ : upper link angular position.

$x_{2}=\theta_{2}$ : lower link angular position.

$x_{3}=\dot{\theta}_{1}$ : upper link angular velocity.

$x_{4}=\dot{\theta}_{2}:$ lower link angular velocity.

so that

$\dot{x}_{1}=x_{3}$

$\dot{x}_{2}=x_{4}$

$\dot{x}_{3}=\frac{K_{4}\left(\tau_{1}-K_{2} x_{4}^{2} \sin \left(x_{1}-x_{2}\right)-K_{3} \sin \left(x_{1}\right)\right)-K_{2} \cos \left(x_{1}-x_{2}\right)\left(\tau_{2}+K_{2} x_{3}^{2} \sin \left(x_{1}-x_{2}\right)-K_{5} \sin \left(x_{2}\right)\right)}{\left(K_{1} K_{4}-K_{2}^{2} \cos \left(x_{1}-x_{2}\right)^{2}\right)}$

$\dot{x}_{4}=\frac{K_{1}\left(\tau_{2}+K_{2} x_{3}^{2} \sin \left(x_{1}-x_{2}\right)-K_{5} \sin \left(x_{2}\right)\right)-K_{2} \cos \left(x_{1}-x_{2}\right)\left(\tau_{1}-K_{2} x_{4}^{2} \sin \left(x_{1}-x_{2}\right)-K_{3} \sin \left(x_{1}\right)\right)}{\left(K_{1} K_{4}-K_{2}^{2} \cos \left(x_{1}-x_{2}\right)^{2}\right)}$ 
The outputs are:

$y_{1}=\theta_{1}$ : upper link angular position.

$y_{2}=\theta_{2}:$ lower link angular position.

And the inputs are:

$u_{1}=\tau_{1}$ : upper-link actuator torque exerted.

$u_{2}=\tau_{2}$ : lower-link actuator torque exerted.

The equations can be rewritten in the following compact form [2]:

$$
\dot{x}(t)=f(x)+g(x) u(t)
$$

where $x \in R^{n}$ is the state, $u \in R^{m}$ denotes the control input, where $n=4$ and $m=2, f(x)$ and $g(x)$ are sufficiently smooth vector fields, and the output equation is:

$$
y(t)=h(x)
$$

By linearizing equations (8) to (11) using Jacobeans' method and with the following initial condition:

$\left(x_{1}, x_{3}\right)=\left(\theta_{1}, \theta_{2}\right)=\left(10^{\circ}, 20^{\circ}\right),\left(\dot{x}_{1}, \dot{x}_{2}\right)=\left(\dot{\theta}_{1}\right.$, $\left.\dot{\theta}_{2}\right)=(0.3,0.4) \mathrm{rad} / \mathrm{s}$ and $\left(\tau_{1}, \tau_{2}\right)=(0.5,0.5)$ N.m.

The resulting state space representation for the system is [2]:

$$
\begin{gathered}
\dot{x}(t)=A x(t)+B u(t) \\
y(t)=C x(t)+D u(t)
\end{gathered}
$$

where $A$ and $B$ are obtained as:

$$
\begin{gathered}
A= \\
{\left[\begin{array}{cccc}
0 & 0 & 1 & 0 \\
0 & 0 & 0 & 1 \\
-19.8873 & 3.8075 & -0.2155 & -0.2039 \\
18.5281 & -22.2111 & 0.6116 & 0.2874
\end{array}\right]} \\
B=\left[\begin{array}{cc}
0 & 0 \\
0 & 0 \\
49.2616 & -69.4362 \\
-69.4362 & 197.0466
\end{array}\right] \\
C=\left[\begin{array}{llll}
1 & 0 & 0 & 0 \\
0 & 1 & 0 & 0
\end{array}\right], D=\left[\begin{array}{ll}
0 & 0 \\
0 & 0
\end{array}\right]
\end{gathered}
$$

The parameters of human swing leg system (i.e. support human leg or humanoid robot leg) are given in Table 1.

Table 1: The parameters of human swing leg(i.e. support human leg or humanoid robot leg) [6].

\begin{tabular}{|c|c|c|}
\hline parameter & value & unit \\
\hline$m_{1,} m_{2}$ & 0.1 & $k g$ \\
\hline$l_{1} l_{2}$ & 0.55 & $m$ \\
\hline$g$ & 9.81 & $m /$ seconds $^{2}$ \\
\hline
\end{tabular}

\section{Controller Design}

Sliding Mode Control is considered as one of the effective nonlinear robust control approaches. It has the ability to compensate the system uncertainty. The SMC includes two modes. These modes are the reaching mode and the sliding mode. A reaching control law is applied, in the reaching mode, to drive the states of system to the sliding line rapidly. When states of the system are on the sliding line, an equivalent control law is applied to drive the states of system, along the sliding line, to the origin and the system is said to be in the sliding mode [7, 8]. Figure 3 illustrates the reaching phase and the sliding surface in the SMC design.

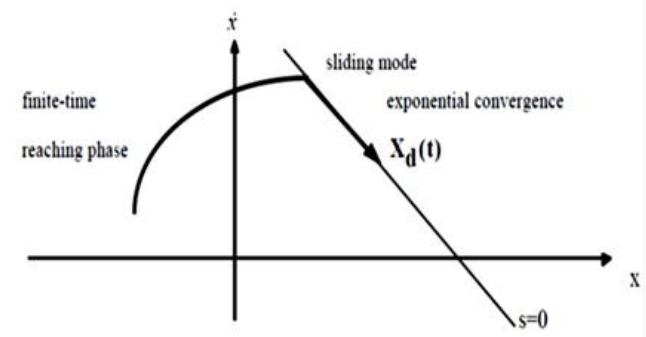

Figure 3: Two steps of SMC design [8].

SMC design consists of two steps, the first step is to find the sliding mode surface, and the second step is to design the control input to force the system trajectory toward the sliding mode surface [9]. If a system has $m$ inputs, there are $m$ hyperplanes, for the human swing leg system which has 2 inputs so there are 2 hyperplanes; these hyperplanes are defined as [9- 11]:

$$
S(t)=G x(t)
$$

where $G$ is $m \times n$ matrix which represent the gain to inform sliding surface. Differentiating equation (17) and substitute equation (14) in the resulting equation, yields:

$$
\dot{S}(t)=G(A x(t)+B u(t))
$$

The sliding mode control law is:

$$
u(t)=u_{e q}(t)+u_{s}(t)
$$

where $u_{e q}(t)$ is an equivalent control input and $u_{s}(t)$ is discontinuous control input. The vector $S(t)=0$ represents the intersection of all $m$ sliding hyperplanes passing through the origin of the state space. The equivalent control input 
$u_{e q}(t)$, corresponds to $\dot{S}(t)=0$. Therefore, from equation (18),

$$
u_{e q}=-(G B)^{-1} G A x
$$

and the matrix $G B$ has been assumed to be nonsingular. The reaching condition for each hyperplanes are $S \dot{S}<0$ so that

$$
\dot{S}=\operatorname{diag}(\eta) \operatorname{sgn}(\mathrm{S})
$$

the discontinuous control input $u_{s}$ have the following form:

$$
u_{s}=-(G B)^{-1} \operatorname{diag}(\eta) \operatorname{sgn}(\mathrm{S})
$$

substitute equations (20) and (22) in (19) so the control law becomes:

$u=-(G B)^{-1}\{G A x+\operatorname{diag}(\eta) \operatorname{sgn}(\mathrm{S})\}$

where $\operatorname{diag}(\eta)$ is a diagonal matrix with the $i$ th diagonal element equal to a positive number $\eta_{i}$.

The $G$ matrix will be computed by minimizing a quadratic objective function involving the state vector and the effective input, that is, by solving a liner quadratic (LQ) problem. This approach is called the Optimal Sliding Mode controller where the objective function to be minimized is [9],

$$
J=\int_{0}^{\infty}\left(x^{T} Q x\right) d t
$$

For the LQ control, it is necessary to have the input term in the quadratic objective function. Here, the input term is not present in the objective function in equation (24), and the constraints are that the system is on the intersection on $m$ sliding hyperplanes.

The matrix $G$ is not specified a priori and will come out as a solution to the problem. Using the similarity transformation

where

$$
q=H x
$$

$$
H=\left[\begin{array}{ll}
N & B
\end{array}\right]^{T}
$$

and the columns of the $n \times(n-m)$ matrix , are composed of basis vectors of the null space of $B^{T}$. From equations (14) and (25) yield,

where

$$
\dot{q}=\mathcal{A} q+\mathcal{B} u
$$

and

$$
\mathcal{A}=H A H^{-1}
$$

$$
\mathcal{B}=H B
$$

because of the special structure of the matrix $H$, the first $(n-m)$ rows of $\mathcal{B}$ turn out to be zeroes. Hence, the vector $q$ is decomposed as follows:

$$
q=\left[\begin{array}{l}
q_{1} \\
q_{2}
\end{array}\right]
$$

where $q_{1}$ and $q_{2}$ are $(n-m)$ and $m-$ dimensional vectors, respectively. Partitioning equations (27) yields,

$$
\left[\begin{array}{l}
\dot{q}_{1} \\
\dot{q}_{2}
\end{array}\right]=\left[\begin{array}{ll}
\mathcal{A}_{11} & \mathcal{A}_{12} \\
\mathcal{A}_{21} & \mathcal{A}_{22}
\end{array}\right]\left[\begin{array}{l}
q_{1} \\
q_{2}
\end{array}\right]+\left[\begin{array}{c}
0 \\
\mathcal{B}_{1}
\end{array}\right] u
$$

substituting equation (27) in equation (24) yields,

where,

$$
J=\int_{0}^{\infty}\left(q^{T} Q_{q} q\right) d t
$$

$$
Q_{q}=\left(H^{-1}\right)^{T} Q H^{-1}
$$

If $Q=Q^{T} \geq 0, Q_{q}=Q_{q}{ }^{T} \geq 0$ then because the signs of eigenvalues are preserved under congruence transformation. Partition $Q_{q}$ to conform to the partition of $q$ in equation (26) as follows:

$$
Q_{q}=\left[\begin{array}{cc}
Q_{r} & N \\
N^{T} & R
\end{array}\right]
$$

substituting equations (30) and (34) in equation (32),

$$
J=\int_{0}^{\infty}\left(q_{1}^{T} Q_{r} q_{1}+2 q_{1}{ }^{T} N q_{2}+q_{2}{ }^{T} R q_{2}\right) d t
$$

The matrix $Q$ is selected by trial and error to be,

$$
Q=10^{5}\left[\begin{array}{llll}
1 & 0 & 0 & 0 \\
0 & 1 & 0 & 0 \\
0 & 0 & 1 & 0 \\
0 & 0 & 0 & 1
\end{array}\right]
$$

Then from the selected $Q$ matrix and according to equations (33) and (34) the matrices $Q_{q}, Q_{r}, R$ and $N$ will be obtained as follows:

$$
\begin{gathered}
Q_{q}=10^{4}\left[\begin{array}{cccc}
10 & 0 & 0 & 0 \\
0 & 10 & 0 & 0 \\
0 & 0 & 0.0183 & 0.0072 \\
0 & 0 & 0.0072 & 0.0030
\end{array}\right], \\
Q_{r}=10^{4}\left[\begin{array}{cc}
10 & 0 \\
0 & 10
\end{array}\right], N=\left[\begin{array}{ll}
0 & 0 \\
0 & 0
\end{array}\right] \\
R=10^{4}\left[\begin{array}{ll}
0.0183 & 0.0072 \\
0.0072 & 0.0030
\end{array}\right]
\end{gathered}
$$

For $S=0$, the $(n-m)$ dimensional dynamics is represented by

$$
\dot{q}_{1}=\mathcal{A}_{11} q_{1}+\mathcal{A}_{12} q_{1}
$$


then sliding hyperplanes can be described as

$$
S=K q_{1}+q_{2}
$$

from equations (17) and (38),

$$
G=\left[\begin{array}{ll}
K & I m
\end{array}\right]^{T} H
$$

Equation (40) indicates that the matrix $G$ can be determined via the matrix $K$. The first objective behind the choice of the matrix $G$ or the matrix $K$ is to ensure the system stability on the intersection of all hyperplanes. Equations (34) and (35) constitute a standard LQ problem provided $R>0$. If $Q$ is chosen to be positive definite, $R$ is guaranteed to be positive definite. In general, $R$ is not guaranteed to be positive definite if $Q$ is positive semi-definite. If $R$ does not turn out to be positive definite, it has to be arbitrarily chosen to be a positive definite matrix. In this case, a new $Q$ will be defined according to equation (34). The gain matrix $K$ for the minimum value of $J$ is $[9,10]$ :

$$
K=R^{-1}\left(\mathcal{A}_{12}{ }^{T} P+N^{T}\right)
$$

where $P$ matrix is obtained from the solution of the Riccati equation:

$$
\begin{aligned}
& P\left(\mathcal{A}_{11}-\mathcal{A}_{12} R^{-1} N^{T}\right)+\left(\mathcal{A}_{11}-\right. \\
& \left.\mathcal{A}_{12} R^{-1} N^{T}\right)^{T} P-P \mathcal{A}_{12} R^{-1} \mathcal{A}_{12}{ }^{T} P+Q_{q}- \\
& N R^{-1} N^{T}=0
\end{aligned}
$$

where

$$
P=\left[\begin{array}{cc}
10 & 0 \\
0 & 10
\end{array}\right]
$$

For tracking, $x_{\mathrm{d}}$ is defined as a desired set point and $e$ is an error signal and the difference between $x$ and $x_{\mathrm{d}}$ is:

$$
e=x-x_{d}
$$

by repeating the same procedure that used for stabilization, substituting equation (44) in equation (17) yields,

$$
S=G e
$$

so that,

$$
\dot{S}=G \dot{e}=G\left(A e+B u+A x_{\mathrm{d}}-\dot{x}_{\mathrm{d}}\right)
$$

if $S=0$,then the equivalent control input $u_{e q}$ becomes,

$$
u_{e q}=-(G B)^{-1} G\left(A e+A x_{\mathrm{d}}-\dot{x}_{\mathrm{d}}\right)
$$

if the reaching condition is $S \dot{S}<0$, then the hyperplanes are satisfied by the following control law:

$$
\begin{gathered}
u=-(G B)^{-1} G\left(A e+A x_{\mathrm{d}}-\dot{x}_{\mathrm{d}}\right)- \\
(G B)^{-1} \operatorname{diag}(\eta) \operatorname{sgn}(\mathrm{S})
\end{gathered}
$$

the resulting state feedback gain matrix and diagonal matrix are :

$$
\begin{aligned}
G= & {\left[\begin{array}{cccc}
0.4926 & -0.6944 & 0.4926 & -0.6944 \\
-0.6944 & 1.9705 & -0.6944 & 1.9705
\end{array}\right], } \\
& \operatorname{diag}(\boldsymbol{\eta})=\left[\begin{array}{ll}
\mathbf{1} & \mathbf{0} \\
\mathbf{0} & \mathbf{1}
\end{array}\right]
\end{aligned}
$$

\section{Related Work}

Many researches have been carried out for controlling the swing leg system by various control methods [1]. In 2004, Ono et al [12] and in 2007, Huang et al [13] The lost energy in joint self-impact stopper was obtained. To restore the lost energy, a torque was applied to the hip joint; that torque was obtained by a proportional controller. That approach was used for stabilization and not for tracking of the swing leg system. In 2010, Dallali et al [14] presented a comparison between PID and Linear Quadratic Regulator (LQR) controllers. The PID controller has been used as a basis for quantification of robustness and performance of humanoid robots. A better robustness was obtained from the LQR controller. The test was done on the robot leg from $-11.50^{\circ}$ to $11.50^{\circ}$ and obtained high control action about 30 N.m. In 2014, Gregg et al [15] implemented virtual constraints that unify the stance period, coordinate ankle and knee control, and accommodate clinically meaningful conditions on a powered prosthetic leg. The saturate prosthesis torques at 80 N.m to simulate the torque limit of the experimental prosthesis. In 2015, Bazargan-Lari et al [6] proposed a nonlinear intelligent controller using Adaptive Neural Network control for human swing leg hip and knee joints. The results were obtained for the joints angular velocity with an acceptable maximum error of about $0.15 \%$ for the hip joint and $0.35 \%$ for the knee joint.

\section{$5 \quad$ Result and Discussion}

Figure 4 illustrates the behavior of the human swing leg system without controller. In this Figure, the hip and knee angular positions are explained. This system inherently unstable, for open loop because it has roots in the right hand side of $s$-plane, and marginally stable for closed loop with high oscillation. The system has the following eigenvalues $\{0.0463 \pm 5.4358 \mathrm{i}$, $0.0643 \pm 3.6555 i\}$ for the open loop and has the 
following eigenvalues $\quad\{-0.0149 \pm$ $15.9877 \mathrm{i},-0.0030 \pm 5.7972 \mathrm{i}\}$ for the closed loop.

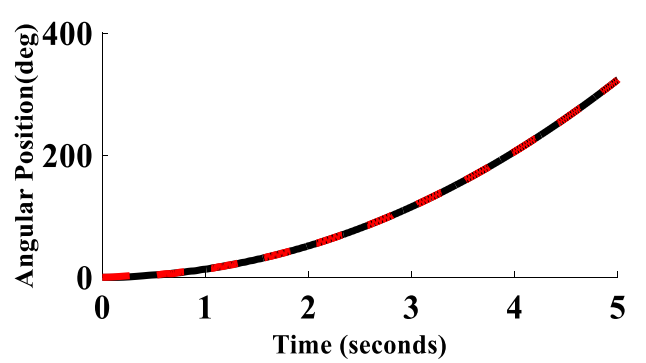

(a)

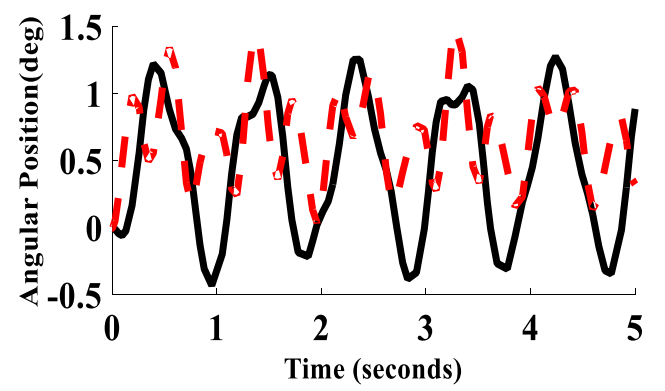

(b)

Figure 4: Step response for hip and knee positions for the human swing leg system without controller for hip (solid line) and knee (dotted line) joints (a) open loop (b) closed loop.

Figure 5 shows the block diagram for the nonlinear human swing leg system with Sliding Mode controller to achieve desired angular positions $\left(\theta_{d 1}, \theta_{d 2}\right)$.

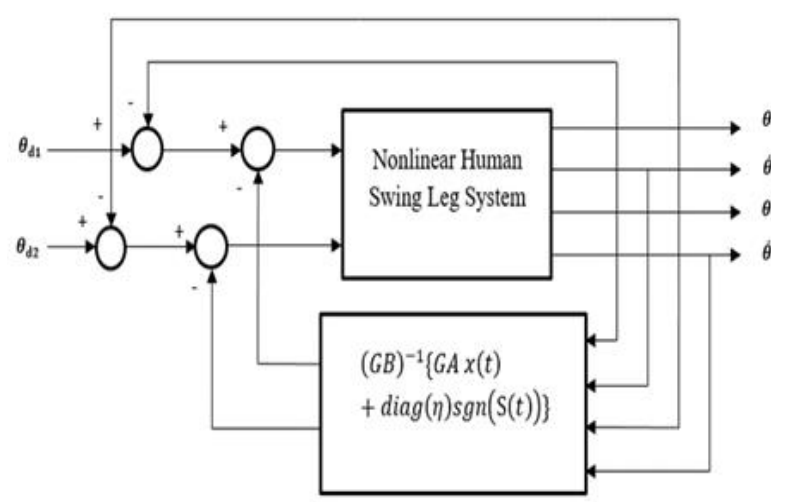

Figure 5: The block diagram for the nonlinear human swing leg system with controller.

Figure 6 shows the Simulink Matlab for the nonlinear human swing leg system with SMC.

Figure 7 shows the time response of the system after applying the designed SMC in case of stabilization. This Figure shows that the achieved settling time is (3.5) seconds. Further, the proposed controller has achieved acceptable control actions.

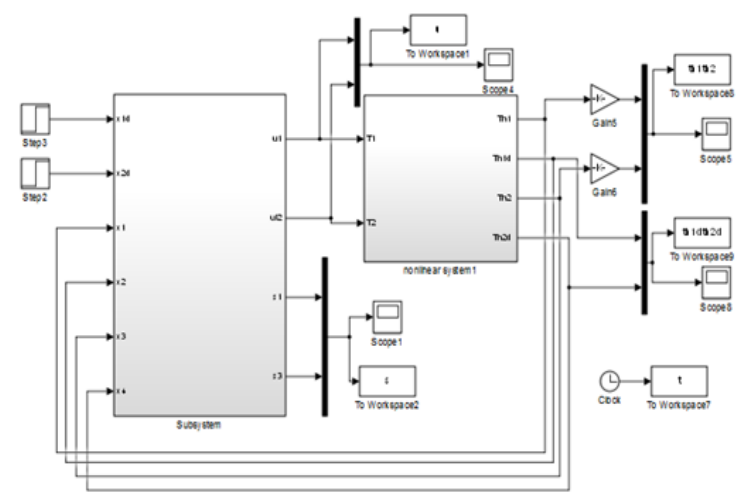

Figure 6: The block diagram and Simulink Matlab for the nonlinear human swing leg system with controller.

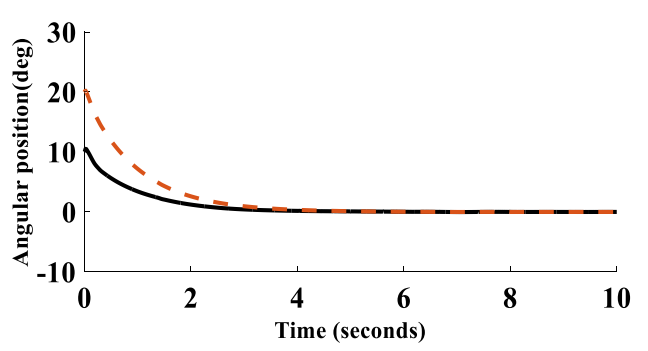

(a)

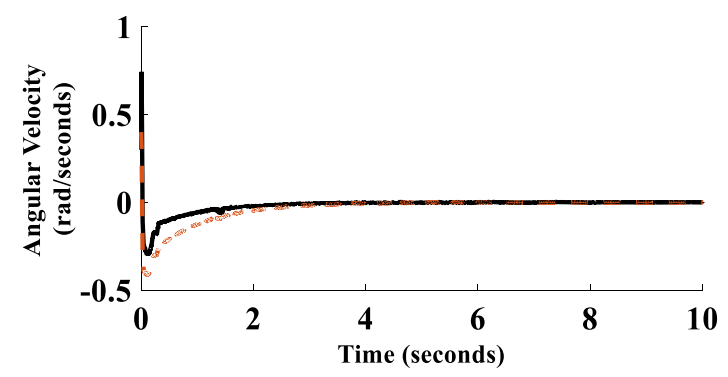

(b)

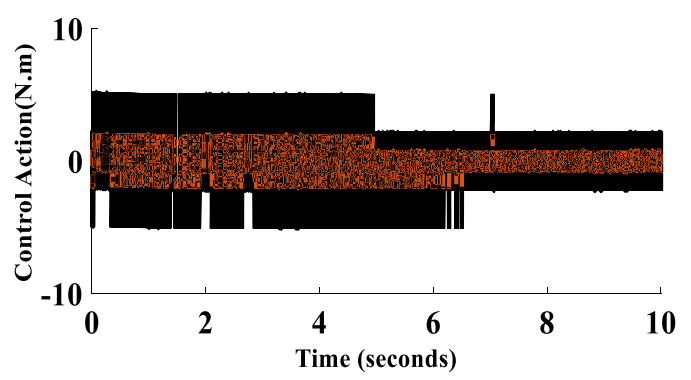

(c)

Figure 7: State trajectories and control actions for the nonlinear system with initial condition $\theta_{1}=$ $10^{\circ}$ and $\theta_{2}=20^{\circ}$ for hip (solid line) and knee (dotted line) joints (a) position (b) velocity (c) control action. 
The ability of the controlled system to track specific trajectories has been shown in Figure 8. The achieved time response specifications are $t_{r}=3.7$ seconds, $t_{s}=4.2$ seconds for hip joint and $t_{r}=3.5$ seconds, $t_{s}=4$ seconds for knee joint.

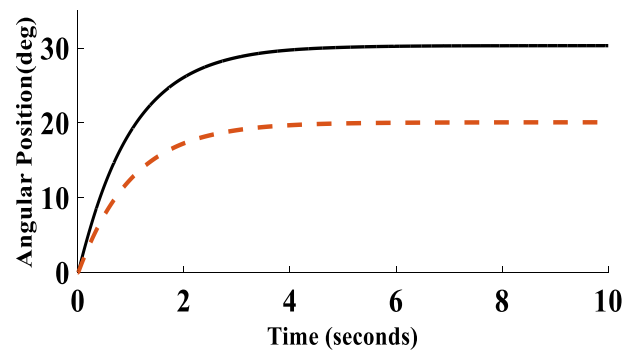

(a)

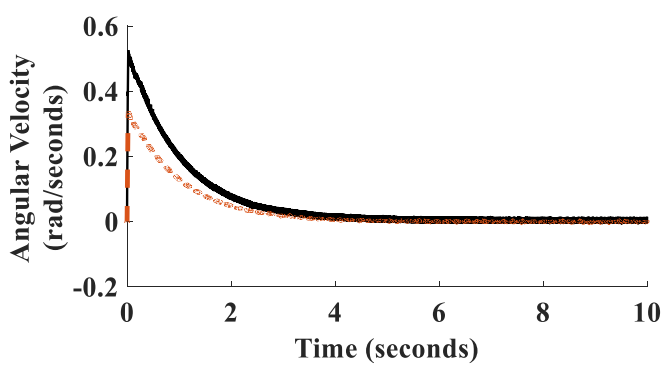

(b)

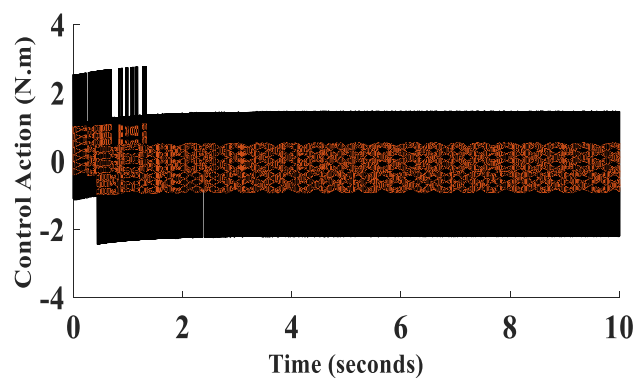

(c)

Figure 8: Time response for the nonlinear human swing leg system with $\theta_{d 1}=20^{\circ}$ and $\theta_{d 2}=30^{\circ}$ for hip (solid line) and knee (dotted line) joints (a) position (b) velocity (c) control action.

To test the robustness of the controlled system, the test is done for the controlled system with $\pm 20 \%$ variation in system parameters. It is obvious from Figure 9 that the proposed controller has a high ability to compensate the system parameters variation and achieve a more desirable time response.

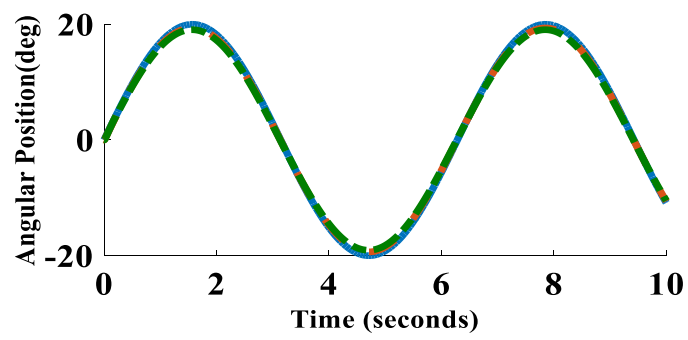

(a)

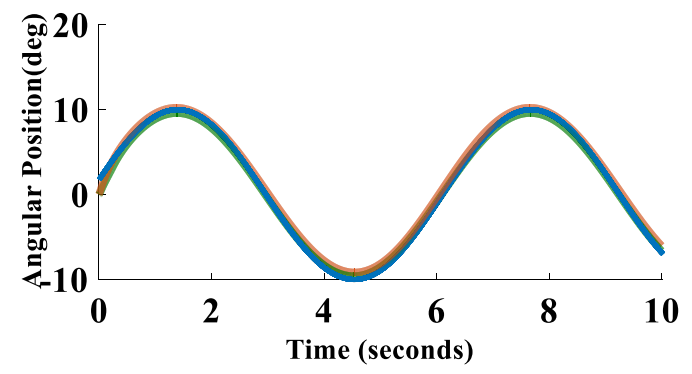

(b)

Figure 9: Time response of the nonlinear human swing leg system with $\pm 20 \%$ variation in system parameters with desired angle (sold line), minimum angle (dotted line) and maximum angle (center line) (a) hip position (b) knee position.

Figure 10 shows the time response of the controlled system with a disturbance. The applied disturbance is $10 \%$ from the reference input and it was applied at $\mathrm{t}=2$ seconds. It is shown that the proposed controller can effectively reject the disturbance.

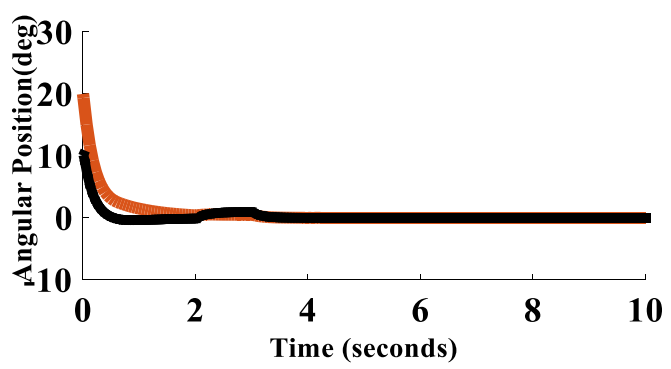

(a)

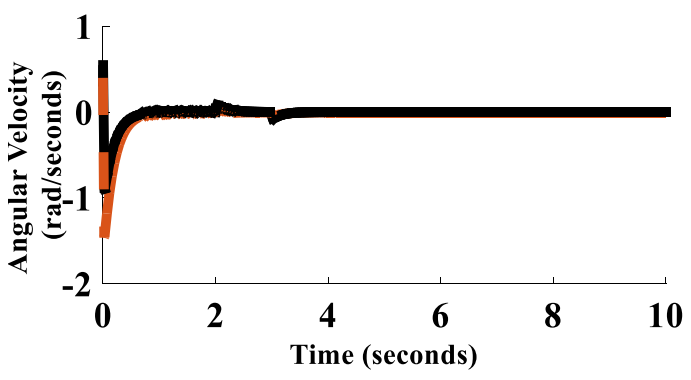

(b)

Figure 10: Disturbance properties of the nonlinear human swing leg system with initial condition $\theta_{1}=10^{\circ}$ and $\theta_{2}=20^{\circ}$ for hip (solid line) and knee (dotted line) joints (a) position (b) velocity. 
The response of the system to sinusoidal signal which represents the ability of the system to track desired trajectories is shown in Figure 11.

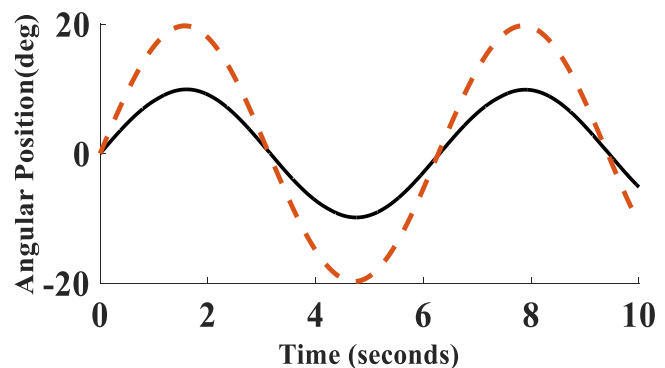

(a)

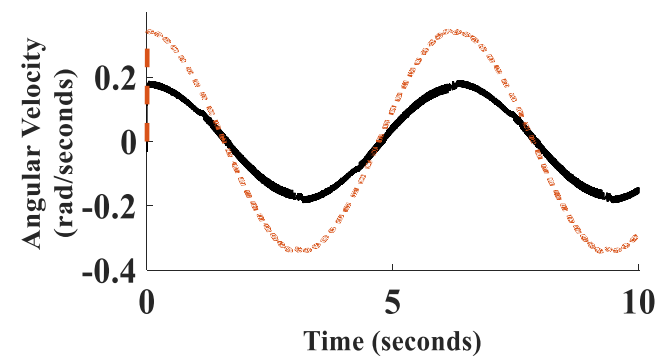

(b)

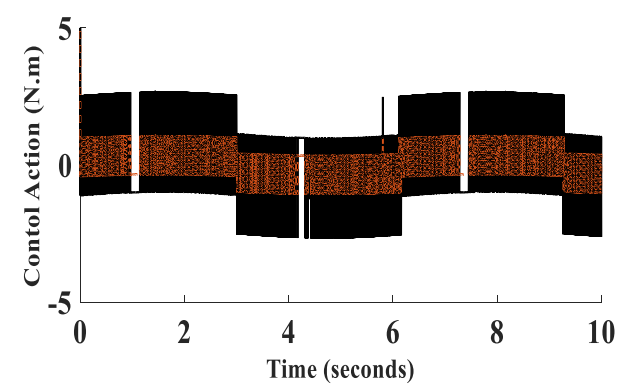

(c)

Figure 11: Time response of the nonlinear human swing leg system to sinusoidal input for hip (solid line) and knee (dotted line) joints (a) position (b) velocity (c) control action.

\section{Conclusions}

In this paper, a sliding mode control (SMC) approach is proposed for the stabilization of uncertain nonlinear a human swing leg system. A sliding mode control is proposed to achieve trajectory tracking of the system. The linearized mathematical model of nonlinear system is derived to facilitate the controller design. And the results show the advantage and applicability of the proposed design methods. Sliding mode control system which is insensitive to uncertainties in system parameters and external disturbances in the whole control process was designed.

\section{References}

[1] Y. Bazargan-Lari, M. Eghtesad, A. R. Khoogar and A. Mohammad-Zadeh, "Tracking Control of a Human Swing Leg Considering Self-Impact Joint Constraint by Feedback Linearization Method", Control Engineering and Applied Informatics (CEAI), Vol.17, pp. 99-110, Romania, 2015.

[2] Y. Bazargan-Lari, A. Gholipour, M. Eghtesad, M. Nouri and A. Sayadkooh, "Dynamics and Control of Locomotion of One Leg Walking as Self-Impact Double Pendulum", International Conference on Control, Instrumentation and Automation (ICCIA), pp.201-206, 2011.

[3] A. Mokhtarian, A. Fattah and S. K. Agrawal, "A passive swing-assistive planar external orthosis for gait training on treadmill", The Brazilian Society of Mechanical Sciences and Engineering, 2014.

[4] M. Singla, L. Shieh, G. Song, L. Xie and Y. Zhang, "A New Optimal Sliding Mode Controller Design Using Scalar Sign Function", ISA Transactions, Elsevier Ltd.,2013

[5] L. Wu, P. Shi and H. Gao, "State Estimation and Sliding-Mode Control of Markovian Jump Singular Systems", IEEE Transactions on Automatic Control, Vol. 55, pp. 1213-1219, 2010.

[6] Y. Bazargan-Lari, M. Eghtesad, A. R. Khoogar and A. Mohammad-Zadeh, "Adaptive Neural Network Control of a Human Swing Leg as a Double-Pendulum Considering Self-Impact Joint Constraint", Transactions of the Canadian Society for Mechanical Engineering, Vol. 39, pp. 201219, 2015.

[7] V. Utkin, J. Guldner, J. Shi, "Sliding Mode Control in Electro-Mechanical Systems", 2nd ed., CRC Press is an imprint of the Taylor \& Francis Group, Boca Raton, London, New York, pp. 10- 20, 2009.

[8] Y. Shtessel, C. Edwards, L. Fridman, A. Levant, "Sliding Mode Control and Observation", Springer Science and Business Media (birkhauser), New York, pp. 3-10, 2014.

[9] A. Sinha, "Linear Systems Optimal and Robust Control", CRC Press is an imprint of Taylor and Francis Group, an Informa business, London, New York, pp. 314-317, 2007. 
[10] N. Munro, "Sliding Mode Control in Engineering”, Marcel Dekker, Inc., United Stated, America, pp. 29-63, 2002.

[11] R. Desai and H. Geyer, "Robust Swing Leg Placement Under Large Disturbances", International Conference, Robotics and Biomimetics, IEEE, Guangzhou, China, pp.265-270, 2012.

[12] K. Ono, T. Furuichi and R. Takahashi, "Self-Excited Walking of a Biped Mechanism with Feet", The International Journal of Robotics Research, Vol. 23, pp.55-68, 2004.

[13] Q. Huang, T. Hase, and K. Ono, "Passive/active unified dynamic walking for biped locomotion", In Robotics and
Biomimetic, ROBIO 2007. IEEE International Conference, pp. 964-971, 2007.

[14] H. Dallali, G. A. Medrano-Cerda and M. Brown,"A Comparison of Multivariable and Decentralized Control Strategies for Robust Humanoid Walking", CICADA project, University of Manchester, 2010.

[15] R. D. Gregg, T. Lenzi, L. J. Hargrove and J. W. Sensinger, "Virtual Constraint Control of a Powered Prosthetic Leg from Simulation to Experiments with Transfemoral Amputees", USAMRAA grant, National Institute of Child Health and Human Development (NIH), IEEE, 2014.

\section{تأثير التغذيه الراجعه لمسيطر التحكم الانزلاقي لمنظومه الساق البشريه المتأرجحه}

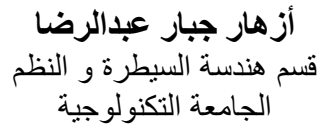

أزهار جبار عبدالرضا

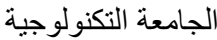

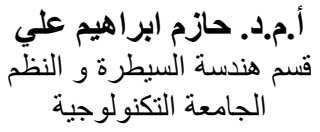

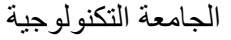

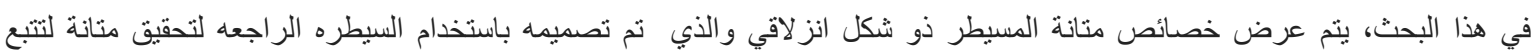

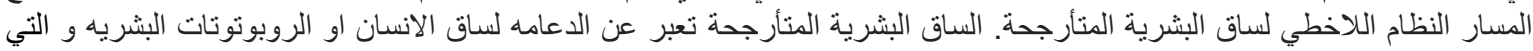

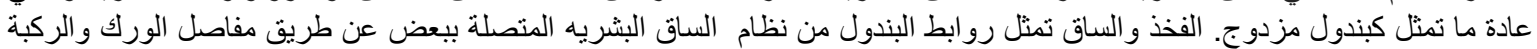

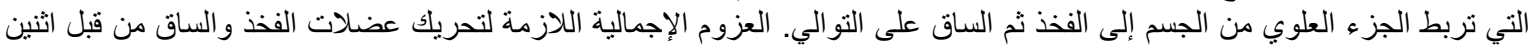

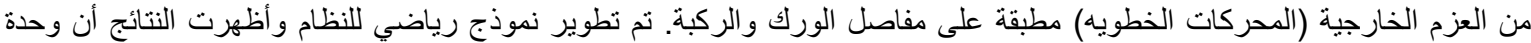
السيطره المقترحة يمكن أن نستقر بقوة النظام وتحقيق مو اصفات زمن من الاستجابة المر غوبة. 